\title{
HYPERSPACES OF A CONTINUUM
}

\author{
BY \\ J. L. KELLEY
}

Introduction. Among the topological invariants of a space $X$ certain spaces have frequently been found valuable. The space of all continuous functions on $X$ and the space of mappings of $X$ into a circle are noteworthy examples. It is the purpose of this paper to study two particular invariant spaces associated with a compact metric continuum $X$; namely, $2^{X}$, which consists of all closed nonvacuous subsets of $X$, and $\mathcal{C}(X)$, which consists of closed connected nonvacuous subsets $\left({ }^{1}\right)$. The aim of this study is twofold. First, we wish to investigate at length the topological properties of the hyperspaces, and, second, to make use of their structure to prove several general theorems.

If $X$ is a compact metric continuum it is known that: $2^{x}$ is Peanian if $X$ is Peanian [7], and conversely [8]; $2^{X}$ is always arcwise connected [1]; $2^{x}$ is the continuous image of the Cantor star [4]; if $X$ is Peanian, each of $2^{X}$ and $\mathcal{C}(X)$ is contractible in itself [9]; and if $X$ is Peanian, $2^{X}$ and $\mathcal{C}(X)$ are absolute retracts $[10]$.

In $\S \S 1-5$ of this paper further topological properties are obtained. In particular: $2^{x}$ has vanishing homology groups of dimension greater than 0 , both hyperspaces have very strong higher local connectivity and connectivity properties-including local $p$-connectedness in the sense of Lefschetz for $p>0$, and, the question of dimension is resolved except for the dimension of $\mathcal{C}(X)$ when $X$ is non-Peanian. All of the results of the preceding paragraph for $2^{X}$ are shown simultaneously for $2^{X}$ and $\mathcal{C}(X)$ in the course of the development.

In $\$ 6$ a characterization of local separating points in terms of $\mathcal{C}(X)$ is obtained and a theorem of G. T. Whyburn deduced. In $\$ 7$ it is shown that for a continuous transformation $f(X)=Y$ we may under certain conditions find $X_{0} \subset X$, with $X_{0}$ closed and of dimension 0 , such that $f\left(X_{0}\right)=Y$. In $\S 8$ this result is utilized in the study of Knaster continua. In order that $X$ be a Knaster continuum it is necessary and sufficient that $\mathcal{C}(X)$ contain a unique arc between every pair of elements. If there exist Knaster continua of dimension greater than 1 then there exist infinite-dimensional Knaster continua.

Presented to the Society in three parts, the first under the title On the hyperspaces of a given space on December 28, 1939; the second under the present title on April 26, 1940; the third under the title $A$ theorem on transformations on December 30,1940; received by the editors May 15, 1941. The major part of the material in this paper was contained in the author's dissertation, University of Virginia, June 1940.

(1) For topologization of these spaces and for definitions of terms used in the introduction see the text. A bibliography is given at the end of the article. Numbers in square brackets refer to the bibliography. 
The author wishes to express his gratitude to Professor G. T. Whyburn for his help and encouragement in the preparation of this paper.

1. Preliminaries. Throughout the following, $X$ will denote a compact metric continuum. The letters $a, b, c$ will stand for elements of $X$. For $a, b \in X, \rho(a, b)$ is the distance from $a$ to $b$. Given a collection $a_{i}, a_{i} \in X$, $\left\{a_{i}\right\}$ denotes the subset of $X$ whose elements are the $a_{i}$. In particular, $\{a\}$ is the subset of $X$ consisting of the one element $a$.

The letters $A, B, C$ stand for closed subsets of $X$. By $2^{x}$ we mean the space of all closed, nonvacuous subsets of $X$ metricized by the Hausdorff metric (that is, $\rho^{1}(A, B)=$ g.l.b. $\{\epsilon\}$ for all $\epsilon$ such that $A \subset V_{\epsilon}(B)$ and $B \subset V_{\epsilon}(A)$, where $V_{\epsilon}(A)$ is the sum of all open $\epsilon$-spheres about points of $A$ [2]). If $A \in 2^{X}$ then $A \subset X$. The closed subspace of $2^{X}$ consisting of subcontinua of $X$ is $C(X)$.

Similarly $2^{2^{X}}$ consists of closed, nonvacuous subsets $\mathcal{A}, \mathcal{B}, \mathcal{C}$ of $2^{X}$, with Hausdorff distance $\rho^{2}$. If $A \in 2^{2^{X}}$ then $A \subset 2^{X}$.

For $A \in 2^{X}$ we define $\phi(A)=\left\{\left\{a_{i}\right\}\right\}, a_{i} \in A$. That is, $\phi(A)$ is the subset of $2^{X}$ consisting of all elements $\{a\}$ of $2^{X}$ where $a \in A$. In particular $\phi(X)$ is the set of all sets $\{a\}$. We always have $\phi(A) \subset 2^{X}$ and $\phi(A) \in 2^{{ }^{X}}$. For any $A \in 2^{X}, \phi(A)$ is isometric with $A$. Similarly, for $A \in 2^{2^{X}}, \phi(\mathcal{A})$ denotes the subset of $2^{{ }^{2} X}$ consisting of elements $\{A\}, A \in \mathcal{C}$. We have $\phi(\mathcal{A}) \subset 2^{2^{X}}$ and in particular $\phi\left(2^{X}\right) \subset 2^{2^{X}}$.

For $A \subset 2^{x}$ we define $\sigma(\mathcal{A})=\sum A$ for all $A \in \mathcal{C}$. For every $A, \sigma(\mathcal{A}) \subset X$. Actually $\sigma$ is a continuous mapping of $2^{2^{X}}$ onto $2^{X}$. Further:

1.1. Lemma. (a) $\sigma$ is a contraction, (b) $\phi \sigma$ is a retraction of $2^{2^{X}}$ onto $\phi\left(2^{X}\right)$.

Proof. First, for $A \in 2^{2}{ }^{X}, \sigma(\mathcal{A})$ is closed. Suppose $a_{i} \in \sigma(\mathcal{A})$, and $\lim a_{i}=a$. Choose $A_{i}, a_{i} \in A_{i} \in \mathcal{C}$. We can suppose $\lim A_{i}=A$. Since $A$ is closed, $A \in \mathcal{C}$ and $a \in A \in \mathcal{C}$. Hence $a \in \sigma(\mathcal{A})$.

Second, suppose $\rho^{1}(\sigma(\mathcal{A}), \sigma(B))=d$. We can choose in one of $\sigma(\mathcal{A}), \sigma(B)$, say in $\sigma(\mathcal{C} A)$, a point $a$ which is at least $d$ distance from every point of $\sigma(B)$. Choose $A, a \in A \in \mathcal{A}$. This set $A$ is then at least $d$ Hausdorff distance from every set $B \in \mathcal{B}$. Hence $\rho^{2}(\mathcal{A}, \mathbb{B}) \geqq d$ and $\sigma$ is shown to be a contraction. That $\sigma$ followed by $\phi$ leaves every element of $\phi\left(2^{X}\right)$ fixed is clear.

1.2. Lemma. If $A$ is a subcontinuum of $2^{X}$ and $\mathcal{A} \cdot \mathcal{C}(X) \neq 0$ then $\sigma(\mathcal{A})$ is a continuum.

Proof. Choose $A \in \mathcal{C} A \cdot \mathcal{C}(X)$. Suppose $\sigma(\mathcal{A})=A_{1}+A_{2}$ is a separation, with $A \subset A_{1}$. Then both the subset $A_{1}$ of $A$ consisting of all elements contained in $A_{1}$ and the subset $A_{2}$ of all elements intersecting $A_{2}$ are closed and nonvacuous. But $A_{1}+A_{2}=\mathcal{A}$, a continuum, and $A_{1} \cdot A_{2}=0$. We then have a contradiction.

It is possible to define $\left({ }^{2}\right)$ a real-valued function $\mu(A)$, continuous on $2^{x}$,

(2) See H. Whitney, Regular families of curves, Annals of Mathematics, (2), vol. 34 (1933), p. 246. 
with the properties:

1.3. If $A \subset B, A \neq B$ then $\mu(A)<\mu(B)$.

1.4. $\mu(X)=1$, and for any $a \in X, \mu(\{a\})=0$.

For convenience, we shall suppose throughout that $\mu(A)$ is a certain fixed function with these properties. Since $2^{X}$ is compact we can further state:

1.5. Lemma. There exists $\eta(\epsilon)>0$ such that if $A, B \in 2^{x}, A \subset B$ and $\mu(B)-\mu(A)<\eta(\epsilon)$ then $\rho^{1}(A, B)<\epsilon$.

2. Segments in $2^{X}$. Let $A_{0}, A_{1} \in 2^{X}$. A segment from $A_{0}$ to $A_{1}$ is a continuous mapping $A_{t}$ of the interval $[0,1]$ into $2^{X}$ which satisfies the two conditions:

2.1. $\mu\left(A_{t}\right)=(1-t) \mu\left(A_{0}\right)+t \mu\left(A_{1}\right)$.

2.2. If $t^{\prime}<t^{\prime \prime}$, then $A_{t^{\prime}} \subset A_{t^{\prime \prime}}$.

2.3. Lemma. Given $A_{0}, A_{1} \in 2^{X}$, there exists a segment from $A_{0}$ to $A_{1}$ if and only if $A_{0} \subset A_{1}$ and every component of $A_{1}$ intersects $A_{0}$.

Proof. First, suppose that $A_{t}$ is a segment from $A_{0}$ to $A_{1}$. If $A_{1}=B_{0}+B_{1}$ is a separation of $A_{1}$ such that $A_{0} \subset B_{0}$, then the subset of $[0,1]$ consisting of all $t$ such that $A_{t} \subset B_{0}$ and the subset defined by $A_{t} \cdot B_{1} \neq 0$ are closed, disjoint and they cover $[0,1]$. Hence $B_{1}=0$.

Second, suppose $A_{0}, A_{1} \in 2^{X}, A_{0} \subset A_{1}$ and every component of $A_{1}$ intersects $A_{0}$. Consider the collection of all sets $A \subset 2^{X}$ which have the two properties:

2.4. If $B \in \mathcal{A}$ then $A_{0} \subset B \subset A_{1}$ and every component of $B$ intersects $A_{0}$.

2.5. If $B_{0}, B_{1} \in \mathcal{A}$ then either $B_{0} \subset B_{1}$ or $B_{0} \supset B_{1}$.

The sum of a monotone family of sets $A$ of this collection is surely a member of the collection. Hence there must exist a member $\mathcal{A}_{0}$ which is saturated with respect to 2.4 and 2.5. Since the closure of $\mathcal{A}_{0}$ also satisfies 2.4 and 2.5, it follows that $A_{0}$ is closed.

We now define for $t, 0 \leqq t \leqq 1, A_{t}$ to be that element of $A_{0}$ if it exists, such that $\mu\left(A_{t}\right)=(1-t) \mu\left(A_{0}\right)+t \mu\left(A_{1}\right)$. By 2.5 we see that $A_{t}$ is $1-1$ and continuity follows from the continuity of the $\mu$ function. Now the proof will be complete if we show that $A_{t}$ is defined for every $t, 0 \leqq t \leqq 1$, or-what is the same-that for $A_{t^{\prime}}, A_{t^{\prime \prime}} \in \mathcal{C} A_{0}, 0 \leqq t^{\prime}<t^{\prime \prime} \leqq 1$, there exists $A \in \mathcal{C} A_{0}$ such that $\mu\left(A_{t^{\prime}}\right)<\mu(A)$ $<\mu\left(A_{t^{\prime \prime}}\right)$. Because of the maximal character of $A_{0}$ it is sufficient to show that there exists some $A \in 2^{X}$ satisfying $A_{t^{\prime}} \subset A \subset A_{t^{\prime \prime}}, \mu\left(A_{t^{\prime}}\right)<\mu(A)<\mu\left(A_{t^{\prime \prime}}\right)$ with every component of $A$ in tersecting $A_{t^{\prime}}$. Choose then $\epsilon>0$ so that $\overline{V_{\epsilon}\left(A_{t^{\prime}}\right)}$ fails to contain $A_{t^{\prime \prime}}$, and let $A$ consist of the components of $A_{t^{\prime \prime}} \cdot \overline{V_{\epsilon}\left(A_{t^{\prime}}\right)}$ which intersect $A_{t^{\prime}}$. Now some component of $A_{t^{\prime \prime}}$ is not contained in $\overline{V_{t}\left(A_{t^{\prime}}\right)}$ and hence $A_{t^{\prime}}$ is a proper subset of $A$, while $A$ is surely a proper subset of $A_{t^{\prime \prime}}$. The required properties follow.

Since any subarc of a segment is, with proper parametrization, a segment, we have 
2.6. Lemma. If $A \in \mathcal{C}(X)$ then every segment with $A$ as beginning is contained in $C(X)$.

The Cantor star is the plane set obtained by joining with a straight line every point of a discontinuum $D$ which lies on the $x$-axis to the point $(0,1)$ on the $y$-axis. Each point of the star can be identified by a point $x \in D$ and a coordinate $y, 0 \leqq y \leqq 1$.

The following theorem has been proved by Mazurkiewicz for $2^{x}$. (See [4] and also [1].)

2.7. Theorem. Each of $2^{X}$ and $\mathcal{C}(X)$ is the continuous image of the Cantor star, and hence arcwise connected $\left({ }^{3}\right)$.

Proof. We first show that the set $\Sigma$ of all segments in $2^{X}$ and the set $\Sigma_{1}$ of all segments with beginning in $\mathcal{C}(X)$ are compact subsets of $\left\{2^{X}\right\} E$, where $E$ is the unit interval. Now $\Sigma$ is an equicontinuous collection of mappings, for, for any segment $A_{t}$, we have $\left|\mu\left(A_{t^{\prime}}\right)-\mu\left(A_{t^{\prime \prime}}\right)\right|=\left|t^{\prime}-t^{\prime \prime}\right|\left(\mu\left(A_{1}\right)-\mu\left(A_{0}\right)\right)$ $\leqq\left|t^{\prime}-t^{\prime \prime}\right|$. Hence by 1.5 , if $\left|t^{\prime}-t^{\prime \prime}\right|<\eta(\epsilon)$ then $\rho^{\prime}\left(A_{t^{\prime}}, A_{t^{\prime \prime}}\right)<\epsilon$. The relations 2.1, 2.2 clearly hold for any limit element and hence $\Sigma$ is compact. That $\Sigma_{1}$ is a closed subset of $\Sigma$ follows from the fact that for a convergent sequence of mappings, the limit of the beginning elements is the beginning element of the limit.

Let $A_{t}(x)$, for $x \in D$, be a continuous mapping of the set $D$ onto $\Sigma$ (or $\Sigma_{1}$ ). Now $A_{t}(x)$ is continuous simultaneously in $x$ and $t$, and since $A_{1}(x)=X$ for any $x \in D$, the mapping $f(x, y)=A_{y}(x)$ is a continuous mapping of the Cantor star onto $2^{x}$ (or $\left.\mathcal{C}(X)\right)$.

3. Contractibility $\left({ }^{4}\right)$. We now have the following lemma

3.1. LеммA. The following properties are equivalent:

(a) $\phi(X)$ is contractible in $2^{X}$.

(b) $2^{X}$ is contractible.

(c) $\mathcal{C}(X)$ is contractible (in itself).

Proof. The proof is in three steps. First, (a) implies (b). If $\phi(X)$ is contractible in $2^{X}$ there exists a continuous mapping $F(a, t)$ of $X \times E$, where $E$ is the unit interval, into $2^{X}$, such that $F(a, 0)=\{a\}, F(a, 1)=$ a constant. Define for $A \in 2^{X}, \mathcal{F}(A, t)=\{F(a, t)\}$ for $a \in A$. Since $F(a, t)$ is a continuous mapping of $X \times E$ into $2^{X}, \mathcal{F}(A, t)$ maps continuously $2^{X} \times E$ into $2^{{ }^{X}}$. The deformation $\sigma(\mathcal{F}(A, t))$ is then continuous and contracts $2^{x}$ in itself.

Second, (b) implies (c). Suppose $2^{X}$ is contractible. There exists a mapping

(3) Actually, in order that a compact metric space $X$ be the continuous image of the Cantor star it is necessary and sufficient that there exist an equicontinuous family of mappings of $E$ into $X$ which includes a map of $E$ covering any pair of points. The proof of this proceeds exactly as that above.

(4) A space $X \subset Y$ is contractible in $Y$ if the identity transformation on $X$ is homotopic to a constant in $Y$. 
$F(A, t)$ of $2^{X} \times E$ into $2^{X}$ such that $F(A, 0)=A$ and $F(A, 1)=$ a constant. Since $2^{X}$ is arcwise connected we can suppose $F(A, 1)=X$ for all $A \in 2^{X}$. Let $\mathcal{F}(A, t)=\left\{F\left(A, t^{\prime}\right)\right\}$ for $0 \leqq t^{\prime} \leqq t$. Now $\mathcal{F}(A, t)$ is surely a continuous mapping of $2^{X} \times E$ into $2^{2^{X}}$. The deformation $G(A, t)=\sigma(\mathcal{F}(A, t))$ will then be continuous and will have the properties: $G(A, 0)=A ; G(A, 1)=X$; if $0 \leqq t^{\prime}<t^{\prime \prime} \leqq 1$, then $G\left(A, t^{\prime}\right) \subset G\left(A, t^{\prime \prime}\right)$. Hence for $A$ fixed, $G\left(A, t^{\prime}\right), 0 \leqq t^{\prime} \leqq t$ defines with proper parametrization, a segment from $G(A, 0)$ to $G(A, t)$. Hence by 2.6 if $A \in \mathcal{C}(X)$ then $G(A, t) \in \mathcal{C}(X)$ for every $t, 0 \leqq t \leqq 1$. Hence $\mathcal{C}(X)$ is contractible.

Third, (c) implies (a). This is obvious.

Remark. It follows from the above arguments that if $2^{X}$ and $\mathcal{C}(X)$ are contractible then the deformation $G(A, t)$ can be chosen to satisfy

$$
G(A+B, t)=G(A, t)+G(B, t) .
$$

If $0 \leqq t^{\prime}<t^{\prime \prime} \leqq 1$ then $G\left(A, t^{\prime}\right) \subset G\left(A, t^{\prime \prime}\right)$.

We shall consider spaces $X$ having the following property:

3.2. For $\epsilon>0$ there exists $\delta(\epsilon)>0$ such that if $a, b \in X, \rho(a, b)<\delta(\epsilon)$ and $a \in A \in \mathcal{C}(X)$, then there exists $B, b \in B \in \mathcal{C}(X)$ with $\rho^{1}(A, B)<\epsilon$.

As a generalization of a theorem of Wojdyslawski (see [9]) we prove

3.3 Theorem. If $X$ has the property of 3.2 then $2^{X}$ and $\mathcal{C}(X)$ are contractible.

Proof. In view of 3.1 it is sufficient to show that $\phi(X)$ is contractible in $2^{X}$. We define now a mapping of $X \times E$ into $2^{{ }^{2} X}$ as follows: $\mathcal{F}(a, t)=\{A\}$ where $a \in A \in \mathcal{C}(X)$ and $\mu(A)=t$. Now for $G(a, t)=\sigma(\mathcal{F}(a, t))$ we have $G(a, 0)=\{a\}$ and $G(a, 1)=X$. Hence the proof reduces to showing the continuity of $\mathcal{F}(a, t)$.

First, for $a \in X$ we show uniform continuity in $t$. Suppose $0 \leqq t^{\prime} \leqq t^{\prime \prime} \leqq 1$. Then from 2.3 we see that for each $A_{1} \in \mathcal{F}\left(a, t^{\prime}\right)$ there exists $A_{2} \in \mathcal{F}\left(a, t^{\prime \prime}\right)$ such that $A_{2} \supset A_{1}$, and similarly, given $A_{2} \in \mathcal{F}\left(a, t^{\prime \prime}\right)$ we can find some $A_{1} \in \mathcal{F}\left(a, t^{\prime}\right)$ with $A_{2} \supset A_{1}$. Hence if $\left|t^{\prime}-t^{\prime \prime}\right|<\eta(\epsilon)$ of 1.5 then every element of each of $\mathcal{F}\left(a, t^{\prime}\right)$ and $\mathcal{F}\left(a, t^{\prime \prime}\right)$ is within $\epsilon$ of some element of the other and $\rho^{2}\left(\mathcal{F}\left(a, t^{\prime}\right)\right.$, $\left.\mathcal{F}\left(a, t^{\prime \prime}\right)\right)<\epsilon$.

Finally, if $t$ is fixed $\mathcal{F}(a, t)$ is continuous in $a$. If $a$ and $b$ are near and $A \in \mathcal{F}(a, t)$ then by 3.2 we can choose $B$ near $A, b \in B \in \mathcal{C}(X)$. Now $\mu(B)$ is near $\mu(A)$. If $\mu(B)>\mu(A)$ we can choose $B_{1}$ on a segment from $\{b\}$ to $B, B_{1}$ near $A$ (see 1.5) with $\mu\left(B_{1}\right)=\mu(A)$. If $\mu(B)<\mu(A)$ we can choose $B_{1}$ on a segment from $B$ to $X$, with $\mu\left(B_{1}\right)=\mu(A)$. In either case we find $B_{1}$ near $A$, $B_{1} \in \mathcal{F}(b, t)$, and continuity is demonstrated.

Examples. Let $X$ be the curve in the $x y$-plane defined by

$$
\begin{aligned}
& y=\sin \frac{1}{x}, \quad \text { for } 0<x \leqq 1, \\
& -1 \leqq y \leqq 1, \quad \text { for } \quad x=0 \text {. }
\end{aligned}
$$


It is easy to verify that condition 3.2 is satisfied for $X$ and hence $2^{X}$ and $\mathcal{C}(X)$ are contractible.

If we add to $X$ the interval

$$
1 \leqq y \leqq \frac{3}{2}, \quad \text { for } \quad x=0
$$

then 3.2 is not satisfied for the curve $X_{1}$ so obtained. Nevertheless, since $X_{1}$ can be deformed into $X, 2^{X_{1}}$ and $\mathcal{C}\left(X_{1}\right)$ are contractible. This shows that condition 3.2 is sufficient without being necessary.

If we now add to $X_{1}$ the points

$$
y=\frac{1}{2}+\sin \frac{1}{x}, \quad \text { for }-1 \leqq x \leqq 0,
$$

we obtain a curve $X_{2}$ for which $2^{X_{2}}$ and $\mathcal{C}\left(X_{2}\right)$ fail to be contractible. If $\mathcal{C}\left(X_{2}\right)$ were contractible we could suppose the deformation $F(A, t)$ satisfied the condition: If $0 \leqq t^{\prime}<t^{\prime \prime} \leqq 1$ then $F\left(A, t^{\prime}\right) \subset F\left(A, t^{\prime \prime}\right)$. If $a \in X_{2}$ and $a$ has a positive $x$-coordinate, there will exist $t_{0}$ such that $F\left(a, t_{0}\right) \subset X$ and $F\left(a, t_{0}\right)$ contains the interval $-1 \leqq y \leqq 1$ for $x=0$. If $b \in X_{2}$ has a negative $x$-coordinate, every continuum containing $b$ is at least one-half unit from $F\left(A, t_{0}\right)$. But $a$ and $b$ can be chosen arbitrarily close, and we have a contradiction.

\subsection{Theorem. The space $2^{x}$ is acyclic in all dimensions.}

Proof. Suppose $Z$ is a $\delta$-cycle in $2^{X}$, that is, an abstract cycle with vertices in $2^{x}$, with the diameter of every simplex less than or equal to $\delta$. For $A \in 2^{X}$ let $F(A)$ be the set of points in $X$ each of which is at most $\delta$ distance from some point of $A$. Now if $\rho^{1}(A, B) \leqq \delta$, then $\rho^{1}(F(A), F(B)) \leqq \delta$, for every point of $F(A)$ is at most $\delta$ distance from some point of $A$, and this point belongs to $F(B)$. Hence if we map each vertex $A_{i}$ of $Z$ into $F\left(A_{i}\right)$ we obtain a $\delta$-cycle $Z_{1}$ with each vertex at most $\delta$ from the corresponding vertex of $Z$. But from the definition of $F$ it follows that there is an integer $n$ such that the $n$th iteration of $F$ carries every $A \in 2^{X}$ into $X$. Hence $Z$ is $3 \delta$ homologous to a cycle on $X \in 2^{X}$. The theorem follows.

Remark. In case $X$ satisfies the condition 3.2 then the preceding theorem as well as a similar theorem for $\mathcal{C}(X)$ is an obvious consequence of 3.3.

Problem. Is $\mathcal{C}(X)$ always acyclic in all dimensions?

4. Local connectedness and retraction properties. Before proceeding we note two lemmas:

4.1. Lemma. If $X$ is Peanian then $2^{X}$ and $\mathcal{C}(X)$ are contractible.

Proof. Any Peano continuum surely has the property of 3.2.

4.2. Lemma. If $A$ is a Peanian subset of $2^{X}($ or $\mathcal{C}(X))$ then $A$ is contractible over a subset $\mathbb{B}$ of $2^{X}($ or $\mathcal{C}(X))$ such that diameter $\mathcal{A}=$ diameter $\mathbb{B}$. 
Proof. If $\mathcal{F}(A, t)$ is a function contracting $\phi(\mathcal{A})$ in $\mathcal{C}(\mathcal{A}),(\mathcal{C}(\mathcal{A})$ is a subset of $\left.\mathcal{C}\left(2^{X}\right)\right)$, then $\sigma(\mathcal{F}(A, t))$ contracts $\mathcal{A}$ in $\sigma(\mathcal{C}(\mathcal{A}))$. Further, $\mathcal{C}(\mathcal{A})$ has the same diameter as $\mathcal{A}$, and $\sigma$ is a contraction.

The following local connectivity property implies local $p$-connectedness in the sense of Lefschetz $\left(^{5}\right)$ for $p>0$.

4.3. TheOREM. Let $K$ be a finite complex, $K_{1}$ a subcomplex including all the 1-dimensional simplices of $K$, and $f\left(K_{1}\right) \subset 2^{X}$ (or $\left.\mathcal{C}(X)\right)$ a continuous mapping such that the partial image of any simplex of $K$ is of diameter less than $\epsilon$. Then $f$ may be extended to a mapping of all of $K$ into $2^{X}($ or $\mathcal{C}(X))$ so that the diameter of the image of any simplex is less than $\epsilon$.

Proof. First, let $f\left(S^{n}\right) \subset 2^{X}$ (or $\left.\mathcal{C}(X)\right), n \geqq 1$, be a map of the surface of an $(n+1)$-cell $E^{n+1}$. Then, by $4.2, f$ may be extended to a map of all of $E^{n+1}$ into $2^{X}$ (or $\mathcal{C}(X)$ ), for the image of $S^{n}$ is a Peano continuum. Now let $f\left(K_{1}\right)$ be the mapping given in the lemma. Then $\phi f$ is a map of $K_{1}$ into $\phi\left(2^{X}\right) \subset \mathcal{C}\left(2^{X}\right)$. We can now extend $f$ to all of each 2 -simplex $x^{2}$ of $K$ so that $x^{2}$ maps into $\mathcal{C}\left(f\left(x^{2} \cdot K_{1}\right)\right)$. Repeating this process, one dimension at a time, we arrive at a mapping $\bar{f}$ of all of $K$, identical with $\phi f$ on $K_{1}$, and such that the image of any simplex $x^{n}$ is contained in $\mathcal{C}\left(f\left(x^{n} \cdot K_{1}\right)\right)$. Hence the diameter of $\bar{f}\left(x^{n}\right)$ equals the diameter of $f\left(x^{n} \cdot K_{1}\right)$. Since $\sigma$ is a contraction, we see that $\sigma \bar{f}$ is the required extension of $f$.

We now reprove a theorem of Wojdyslawski (see [10]; also [7] and [8]).

4.4. ThEOREM (Wojdyslawski). The following statements are equivalent:

(a) $X$ is Peanian.

(b) $2^{X}$ is Peanian.

(b') $\mathcal{C}(X)$ is Peanian.

(c) $2^{X}$ is an absolute retract.

$\left(c^{\prime}\right) \mathcal{C}(X)$ is an absolute retract $\left(^{6}\right)$.

Proof. The proof is contained in the following three assertions:

First, (a) implies (b) and ( $\left.b^{\prime}\right)$. Suppose that any two points of $X$ less than $\nu(\epsilon)$ apart can be joined by a continuum of diameter less than $\epsilon$. Then if $A$, $B \in 2^{X}, \rho^{1}(A, B)<\nu(\epsilon)$, the set $C$ consisting of all points which can be joined to $A$ by continua of diameter at most $\epsilon$ has the properties: $\rho^{1}(A, C) \leqq \epsilon, \rho^{1}(B$, $C) \leqq 2 \epsilon, A+B \subset C$ and every component of $C$ intersects both $A$ and $B$. Hence by 2.3 there exist segments $A_{t}$ and $B_{t}$ from $A$ to $C$ and $B$ to $C$, respectively. The continuum $A=\left\{A_{t}\right\}+\left\{B_{t}\right\}, 0 \leqq t \leqq 1$, is of diameter less than or equal

(5) See S. Lefschetz, Topology, American Mathematical Society Colloquium Publications, vol. 12, 1930, p. 91 .

(6) A space $X \subset Y$ is a retract of $Y$ if there exists a continuous transformation $f(Y)=X$ where $f$ is the identity on $X$. The metric separable space $X$ is an absolute retract if it is a retract of every metric space in which it can be imbedded. See K. Borsuk, Sur les rétractes, Fundamenta Mathematicae, vol. 17 (1931), pp. 152-170. 
to $3 \epsilon$, and $A \subset \mathcal{C}(X)$ if $A$ and $B$ belong to $\mathcal{C}(X)$. Hence $2^{X}$ and $\mathcal{C}(X)$ are Peanian.

Second, (a) implies (c) and $\left(c^{\prime}\right)$. Combining the result of the previous paragraph with that of 4.3 we have: If $K$ is a finite complex,$K_{0}$ a subcomplex including all of the vertices of $K$, and if $f\left(K_{0}\right) \subset 2^{X}$ (or $\mathcal{C}(X)$ ) is a mapping such that the partial image under $f$ of any simplex of $K$ is of diameter less than $\nu(\epsilon / 6)$, then $f$ can be extended to a mapping of all of $K$ into $2^{X}$ (or $\mathcal{C}(X)$ ) such that the image of any simplex of $K$ is of diameter at most $\epsilon$. This result, by a characterization of Lefschetz $\left({ }^{7}\right)$, implies that $2^{X}$ and $\mathcal{C}(X)$ are absolute retracts.

Third, either one of (b) or ( $\left.\mathrm{b}^{\prime}\right)$ implies (a). If $a, b \in X$, and $\phi(a)$ and $\phi(b)$ can be joined in $2^{X}$ by a continuum $\mathcal{A}$ of diameter $d$, then by $1.2 \sigma(\mathcal{A})$ is a continuum in $X$ about $a+b$ of diameter at most $d$.

4.5. TheOREM. Let $Y$ be a compact, locally connected subset of a metric space $Z$, and let $f(Y)$ be a continuous mapping of $Y$ into $2^{X}($ or $\mathcal{C}(X))$. Then $f$ can be extended to a continuous mapping of all $Z$ into $2^{X}($ or $\mathcal{C}(X))$.

Proof. The set $f(Y)$ is locally connected, and since each hyperspace is arcwise connected, we can find a Peano continuum $A, f(Y) \subset \mathcal{A}, \mathcal{A}$ in $2^{X}$ or $\mathcal{C}(X)$, respectively. Since $\mathcal{C}(\mathcal{A})$ is an absolute retract we can extend $\left.{ }^{8}\right)$ the transformation $\phi f$ of $Y$ to a mapping $\bar{f}$ of $Z$ into $\mathcal{C}(\mathcal{A})$. The mapping $\sigma \bar{f}$ is then the required extension of $f$.

Remark. Consider any closed subset $\mathcal{A}$ of $2^{x}$ having the property: If $A \in \mathcal{A}$ and if $B \supset A$ and every component of $B$ intersects $A$ then $B \in \mathcal{A}$. All the results of $\S \S 2,3,4$ for $2^{X}$ (except 3.4 ) can be shown by precisely the same reasoning to hold for such a set $\mathcal{A}$. In particular the space $\mathcal{C}_{n}(X)$ consisting of all closed subsets of $X$ having at most $n$ components, and the space $\mathcal{C}^{d}(X)$ consisting of all closed sets of diameter greater than or equal to $d$ have these stated properties of $2^{x}$.

5. Dimension of hyperspaces. Further topological properties are now obtained.

5.1. The space $2^{x}$ always contains the homeomorph of the "fundamental cube."

Proof. Choose $A_{i} \in \mathcal{C}(X)$, a sequence of nondegenerate disjoint continua tending to a point $a \notin A_{i}$ for any $i$. Now each $2^{A_{i}}$ contains a nondegenerate arc $B_{i}$ and $2^{X}$ contains topologically the infinite cartesian product $B_{1} \times B_{2} \times \cdots$. The theorem follows.

If $X$ is Peanian and $A \in \mathcal{C}(X)$ then the order of $A$ in $X$ is the smallest integer $n$ such that there exists within any $V_{\epsilon}(A)$ a neighborhood of $A$ with

(7) Annals of Mathematics, (2), vol. 35 (1934), pp. 118-129.

(8) This is a property of absolute retracts. See Footnote 6. 
boundary consisting of at most $n$ points. If no such integer exists then $A$ is said to be of non-finite order.

5.2. Lemma. If $X$ is Peanian the order of $A$ is finite for every $A \in \mathcal{C}(X)$ if and only if $X$ is a graph.

Proof. We need only show that if $X$ is not a graph, $X$ contains a continuum of nor-finite order. If $X$ contains no point constituting a continuum of non-finite order, $X$ must contain an infinite sequence $a_{i}$ of ramification points, and we can suppose $a_{i} \rightarrow a$. If there exists an arc containing infinitely many of the $a_{i}$ this arc is of non-finite order. Otherwise, we can choose infinitely many arcs $a_{i_{n}} a$, forming a null sequence and with each $a_{i_{n}}$ contained in only one arc of the sequence. Then $\sum_{n} a_{i_{n}} a$ is a continuum of non-finite order.

If $A$ is a closed subset of $X, \mathcal{C}(X, A)$ is the subset of $\mathcal{C}(X)$ consisting of continua which contain $A$. If $A \in \mathcal{C}(X)$ then $A \in \mathcal{C}(X, A)$. Also $\mathcal{C}(X, 0)=C(X)$.

5.3. Lemma. If $X$ is Peanian, then for every $A \in \mathcal{C}(X)$ we have order $A$ $\leqq \operatorname{dim}_{A} \mathcal{C}(X, A)$.

Proof. If $A \in \mathcal{C}(X)$ is of order $n$, then, using the $n$-Bogensatz $\left({ }^{9}\right)$, we can choose arcs $B_{1}, \cdots, B_{n}, B_{i} \cdot A=a_{i}$ and $\left(B_{i}-a_{i}\right)$ a collection of disjoint sets. To each $\left(t_{1}, t_{2}, \cdots, t_{n}\right) \in B_{1} \times B_{2} \times \cdots \times B_{n}$ assign the continuum $A+\sum_{i}^{n} a_{i} t_{i}$. This correspondence is a homeomorphism and the theorem is proved.

5.4. Theorem. If $X$ is Peanian then $\operatorname{dim} \mathcal{C}(X)<\infty$ if and only if $X$ is a linear graph.

Proof. If $\operatorname{dim} \mathcal{C}(X)$ is finite then 5.3 and 5.2 imply that $X$ is a linear graph. The other half of the theorem is contained in the following sharper statement.

5.5. TheOREM. If $X$ is a connected linear graph then

$$
\begin{aligned}
\operatorname{dim} \mathcal{C}(X) & =\max _{A \in \mathcal{C}_{(X)}}(\operatorname{order} A) \\
& =2+\sum(\operatorname{order} a-2),
\end{aligned}
$$

the last summation being extended over all points $a \in X$ such that order $a \geqq 2$.

Proof. Let $A_{1}, A_{2}, \cdots, A_{m}$ be the collection of connected sub-graphs of $X$. With each $A_{i}$ there is associated the collection $A_{i}$ of continua in $X$ for which $A_{i}$ is the maximal sub-graph. Clearly, $\mathcal{C}(X)$ is the sum of the $A_{i}$. If the order of $A_{i}$ is $n$, then there are, say, $m 1$-cells containing a single 0 -cell of $A_{i}$ and $k$ 1-cells containing 20 -cells of $A_{i}$, where $m+2 k=n$. By the argument used in 5.3, we see that $A_{i}$ is homeomorphic with the $F_{\sigma}$-set in $n$-space given by the inequalities $0 \leqq x_{i}<1$ for $i=1, \cdots, n, x_{2 j-1}+x_{2 j}<1$ for $j=1, \cdots, k$. Since $A_{i}$ is an $F_{\sigma}\left({ }^{10}\right)$,

(9) See "n-Bogensatz," K. Menger, Kurventheorie, p. 216.

(10) See "Summensatz," K. Menger, Dimensiontheorie, p. 92. 


$$
\operatorname{dim} \mathcal{C}(X) \leqq \max _{i}\left(\operatorname{dim} A_{i}\right)=\max _{i}\left(\operatorname{order} A_{i}\right) \leqq \max _{\boldsymbol{A} \in \mathfrak{C}_{(X)}}(\operatorname{order} A) .
$$

The other necessary inequality is contained in 5.3.

The equality $\max _{A} \in \mathfrak{e}_{(x)}(\operatorname{order} A)=2+\sum(\operatorname{order} a-2)$ can be obtained by a simple induction argument.

Remark. If $X$ is a linear graph $\mathcal{C}(X)$ is actually a polyhedron. We have also the property: If $X$ is Peanian and $\mathcal{C}(X)$ has finite dimension at every one of its points then $\mathcal{C}(X)$ must have finite dimension.

6. Local separating points. In this section we prove a theorem of G. T. Whyburn.

6.1. Theorem. If $X$ is Peanian, $A$ any closed subset of $X, a \in X-A$, then $a$ is a local separating point of $X$ if and only if $\mathcal{C}(X, A+a)$ contains interior points relative to $\mathcal{C}(X, A)$.

Proof. First, let $a$ be a nonlocal separating point, $a \in X-A$. For $B \in \mathcal{C}(X, A+a)$ and $\epsilon>0$ choose a connected neighborhood $U$ of $a$ of diameter less than $\epsilon$ so that $\bar{U} \cdot A=0$. Choose a neighborhood $V$ of $a, \bar{V} \subset U$, such that $U-V$ is connected. Then $(B+\overline{U-V}) \in \mathcal{C}(X, A)-\mathcal{C}(X, A+a)$ and is at most $\epsilon$ distance from $B$. Hence $\mathcal{C}(X, A)-\mathcal{C}(X, A+a)$ is dense in $\bigodot(X, A)$.

Second, let $a$ be a local separating point of $X$ and $U$ a connected neighborhood of $a$ such that $U-a=U_{1}+U_{2}, \bar{U}_{1} \cdot \bar{U}_{2}=a$. Let $V$ be a connected neighborhood of $a$ with $\bar{V} \subset U$. Choose a continuum $B \supset A+\bar{V}$ and intersecting the boundary of only one of $U_{1}$ and $U_{2}$ in points other than $a$. Any continuum sufficiently near $B$ intersects both $V \cdot U_{1}$ and $V \cdot U_{2}$ and fails to intersect the boundary of one of $U_{1}$ and $U_{2}$ in a point different from $a$. Hence $a$ is a point of this continuum and $B$ is interior to $\mathcal{C}(X, A+a)$ relative to $\mathcal{C}(X, A)$.

Remark. If $X$ is non-Peanian and $a$ is a local separating point then $\mathcal{C}(X, a)$ contains interior points relative to $\mathcal{C}(X)$. The converse is not necessarily true, however.

If $A$ is the null set we have this corollary.

6.2. Corollary. If $X$ is Peanian, $a \in X$ then a is a local separating point if and only if $\mathcal{C}(X, a)$ contains interior points relative to $\mathcal{C}(X)$.

6.3. Theorem (G. T. Whyburn(11)). If $X$ is Peanian and $a_{i} \in X$ is a sequence of nonlocal separating points, then $X^{*}=X-\sum a_{i}$ is connected and locally connected.

- In fact, if $b_{1}, b_{2} \in X^{*}$, and $b_{1}$ and $b_{2}$ can be joined in $X$ by a continuum of diameter less than $\epsilon$ then the same holds in $X^{*}$.

Proof. The set $\prod_{1}^{\infty}\left(\mathcal{C}\left(X, b_{1}+b_{2}\right)-\mathcal{C}\left(X, b_{1}+b_{2}+a_{n}\right)\right)$ is by the theorem of

(11) Semi-closed sets and collections, Duke Mathematical Journal, vol. 2 (1936), pp. 684-690. The above theorem is contained in Theorem 3.2 of the paper cited. I owe this proof to S. Eilenberg. 
Baire, dense in $\mathcal{C}\left(X, b_{1}+b_{2}\right)$, since by 6.1 each set in the product is dense and open in $\mathcal{C}\left(X, b_{1}+b_{2}\right)$. Hence any continuum about $b_{1}+b_{2}$ is the limit of continua about $b_{1}+b_{2}$ in $X^{*}$. The theorem follows.

7. Continuous transformations. Here we show that for a continuous transformation $f(X)=Y$ we may under certain conditions find $X_{0} \subset X$, with $X_{0}$ closed and of dimension 0 , such that $f\left(X_{0}\right)=Y$.

7.1. Lemma. If $f\left(E^{2}\right)=E^{1}$ is a continuous mapping of the unit square onto the unit interval, then there exist two disjoint arcs $a b$ and cd in $E^{2}$, each containing at most one boundary point of $E^{2}$, such that $f(a b+c d)=E^{1}$.

Proof. The interior of $E^{2}$ maps into a connected set which is dense in $E^{1}$. Choose $a \in f^{-1}(0), b \in f^{-1}(2 / 3), c \in f^{-1}(1 / 3), d \in f^{-1}(1)$ so that $b$ and $c$ do not belong to the boundary $E^{2}$. Choose $a b$ and $c d$ disjoint arcs in $E^{2}$ having at most $a$ and $d$ in common with the boundary of $E^{2}$. Then $f(a b) \supset(0,2 / 3)$ and $f(c d) \supset(1 / 3,1)$.

7.2. THEOREM(12). If $f\left(E^{2}\right)=E^{1}$ is a continuous mapping of the unit square onto the unit interval then there exists a closed totally disconnected subset $Z$ of $E^{2}$ such that $f(Z)=E^{1}$.

Proof. Let $A$ be the subset of $2^{E^{2}}$ consisting of all subsets of $E^{2}$ which map onto $E^{1}$ under $f$. Let $A_{\epsilon}$ be the subset of $\mathcal{A}$ consisting of sets having only components of diameter less than $\epsilon$. Clearly $A_{\epsilon}$ is open in $\mathcal{A}$, and we shall show $A_{\epsilon}$ is dense in $\mathcal{A}$. Since a residual set in a complete space is nonvacuous, it will be true that $\prod \mathcal{C} \mathcal{A}_{1 / n} \neq 0$, and any $A \in \prod \mathcal{A}_{1 / n}$ will be a totally disconnected closed set mapping on $E^{1}$.

Suppose $A \in \mathcal{A}$ and $\epsilon>0$ are given. We shall find $B \in A_{\epsilon}, \rho^{1}(A, B)<\epsilon$. Choose a subdivision of $E^{2}$ into closed squares $S_{1}, S_{2}, \cdots, S_{q}$, each of diameter less than $\epsilon / 4$. For each $S_{p}$ which intersects $A$ choose $\operatorname{arcs} a_{p} b_{p}$ and $c_{p} d_{p}$ by 7.1 , each mapping onto $f\left(S_{p}\right)$, and let $B$ be the sum of the arcs so chosen. Since dia $S_{p}<\epsilon / 4, B$ has only components of diameter less than $\epsilon$. Since $B$ intersects those and only those squares $S_{p}$ which are cut by $A$, $\rho^{1}(A, B)<\epsilon$ and $f(B) \supset E^{1}$. Hence $B \in \mathcal{A}_{\epsilon}$ and the proof is complete.

We now obtain a similar theorem with more general space and more special type of transformation. First, consider a transformation $f(X)=Y$ where

7.3. (a) $X$ is compact and metric and $\operatorname{dim} Y<\infty$.

(b) $f$ is monotone and interior $\left.{ }^{13}\right)$.

(c) $\operatorname{dia} f^{-1}(y)>0$ for all $y \in Y$.

(12) I owe this theorem to S. Eilenberg and L. Zippin.

(13) A transformation is monotone if the inverse of every point in the image space is connected. See R. L. Moore, Foundations of Point Set Theory, American Mathematical Society Colloquium Publications, vol. 13, 1932, chap. 5. The term "monotone" is due to C. B. Morrey, American Journal of Mathematics, vol. 57 (1935), pp. 17-50. A transformation is interior if open sets map into open sets. For references see G. T. Whyburn, Duke Mathematical Journal, vol. 3 (1937), pp. 370-381. 
7.4. Lemma. Under the hypothesis of 7.3 for any $A \in 2^{x}$ where $f(A)=Y$ and for any $\epsilon>0$ there exists $B \in 2^{X}$ such that

(a) $\rho^{1}(A, B)<\epsilon$.

(b) $f(B)=Y$.

(c) Every component of $B$ is of diameter less than $\epsilon$.

Proof. It is sufficient to find $B \subset V_{\epsilon}(A)$ and satisfying (b) and (c) since by adding a finite number of points to such a $B$ we may obtain a set within $\epsilon$ of $A$. Let $V_{0}=V_{\epsilon / 2}(A)$. We shall need these three lemmas:

7.5. Lemma. There exists $\tau(\epsilon)>0$ such that $f\left(V_{\epsilon}(x)\right) \supset V_{\tau(\epsilon)}(f(x))$ for every $x \in X$.

7.6. Lemma. There exists $d>0$ such that for any $y \in Y$ there is a component $A_{y}$ of $V_{0} \cdot f^{-1}(y)$ such that dia $A_{y} \geqq d$.

7.7. LemMA. There exists an integer $N$ such that $Y$ allows an arbitrarily fine covering by open sets, $W_{1}, \cdots, W_{m}$ such that at most $N$ of the sets $\bar{W}_{i}$ intersect any given $\bar{W}_{r}$.

The first of these is a simple consequence of interiority, the second follows since dia $f^{-1}(y)>0$ for all $y \in Y$, and the third is true since $Y$ can be imbedded in a finite-dimensional euclidean space.

Let $s=\min [\epsilon / 3, d / 8 N]$ and construct a covering of $Y$ of the type 7.7 with dia $W_{r}<\tau(s)$ for $r=1, \cdots, m$. Let $U_{i}=f^{-1}\left(W_{i}\right)$. Choose $a_{1} \in U_{1} \cdot V_{0}$ and

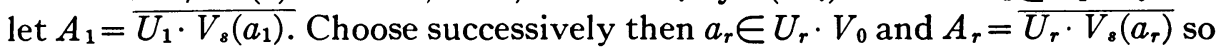
that $A_{r} \cdot A_{i}=0$ for $i<r$. That this is always possible is shown as follows: Choose $y \in W_{r}$ and $A_{y}$ of 7.6. At most $N$ of the sets $A_{1} \cdots, A_{r-1}$ intersect $\bar{U}_{r}$, and each $A_{i}$ is of dia less than $2 s$. If $\sum_{1}^{r-1} V_{s}\left(A_{i} \cdot \bar{U}_{r}\right)$ intersected $V_{s}(a)$ for every $a \in A_{y}$ then $\sum_{1}^{r-1} V_{2 s}\left(V_{s}\left(A_{i} \cdot \bar{U}_{r}\right)\right) \supset A_{y}$ and dia $A_{y} \leqq N \cdot 8 s<d$ which is impossible. Hence it is possible to choose $A_{1}, \cdots, A_{m}$ as prescribed. Finally, $f\left(A_{r}\right) \supset W_{r}$ and $\sum A_{r} \subset V_{\epsilon}(A)$. Let $B=\sum A_{r}$ and the result follows.

7.8. THEOREM. Let $f(X)=Y$ be a monotone interior transformation of a compact metric space $X$ into a set $Y$ of finite dimension. Then there exists a closed totally disconnected subset $X_{0}$ of $X$ mapping onto $Y$ if and only if the set of points on which $f$ is 1-1 is a totally disconnected subset of $Y$.

Proof. First, suppose $f^{-1}(y)$ contains more than a single point for every $y \in Y$. If $A \subset 2^{x}$ is the set of all sets mapping onto $Y$ under $f$, then by 7.4 the subset $\mathcal{A}_{1 / n}$ of sets with components of diameter less than $1 / n$ is dense in $\mathcal{A}$. Any set belonging to the residual set $\prod \mathcal{C} A_{1 / n}$ then satisfies the theorem.

Second, suppose $f^{-1}(y)$ consists of a single point for all $y \in B, B$ a totally disconnected set. Since $f$ is interior, $B$ is closed. Let $V_{n}=V_{1 / n}(B)$ and using the result of the previous paragraph choose $A_{n}$, closed, totally disconnected. and mapping on $\bar{V}_{n}-V_{n+1}$. Then $X_{0}=\sum A_{n}+f^{-1}(B)$ is easily seen to be totally disconnected and maps onto $Y$. 
Finally, if $f$ is $1-1$ on a continuum, it is clearly impossible to find $X_{0}$ satisfying the theorem.

8. Knaster continua. A compact metric continuum is indecomposable if it cannot be written as the sum of two proper subcontinua.

8.1. Lemma. If $X$ is indecomposable and $A_{A B}$ is an arc in $\mathcal{C}(X)$ with $\sigma\left(\mathcal{C} A_{A B}\right)=X$ then $X \in \mathcal{A}_{A B}$.

Proof. Let $C$ be the first element in order from $A$ to $B$ such that $\sigma\left(A_{A C}\right)=X$. For each $C_{1}$ preceding $C$ the continuum $\sigma\left(A_{A C_{1}}\right)$ is contained in the composant about $A$ of $X$, and hence $\sigma\left(A_{C_{1} C}\right)$ contains points both in this composant and in its complement. Thus $\sigma\left(A_{C_{1} C}\right)=X$ for all $C_{1}$ preceding $C$, and therefore $C=X$.

8.2. THEOREM. In order that $X$ be indecomposable it is necessary and sufficient that $\mathcal{C}(X)-X$ fail to be arcwise connected.

Proof. If $X$ is indecomposable then for any arc $A_{A B}$ where $A$ and $B$ lie in different composants of $X$ we have $\sigma\left(\mathcal{A}_{A B}\right)=X$ and hence $X \in \mathcal{A} \mathcal{A}_{A B}$. Thus $\mathcal{C}(X)-X$ is not arcwise connected.

If $X$ is not indecomposable write $X=A_{1}+A_{2}, A_{i} \in \mathcal{C}(X), A_{i} \neq X$ for $i=1$, 2. If $B \in \mathcal{C}(X), B \neq X$, and $a \in B \cdot A_{1} \cdot A_{2}$ then there exists a segment joining $\{a\}$ to $B$, and also segments joining $\{a\}$ to both $A_{1}$ and $A_{2}$. If $B \subset A_{1}$ there is a segment from $B$ to $A_{1}$. In any event $B$ can be joined by an arc to both of $A_{1}$ and $A_{2}$ in $\mathrm{C}(X)-X$ and the theorem is proved.

A compact metric continuum is a Knaster continuum $\left({ }^{14}\right)$ if every subcontinuum is indecomposable. If $X$ is a Knaster continuum and if $A, B \in \mathcal{C}(X)$, then either $A B=0, A \supset B$ or $B \supset A$. Hence:

8.3. Lemma. If $X$ is a Knaster continuum, $A, B \in \mathcal{C}(X), A B \neq 0$ and $\mu(A)=\mu(B)$ then $A=B$.

8.4. THEOREM. The continuum $X$ is a Knaster continuum if and only if $\mathcal{C}(X)$ contains a unique arc between every pair of its elements.

Proof. If $\mathcal{C}(X)$ contains a unique arc between every pair of elements then for any $A \in \mathcal{C}(X), \mathcal{C}(A)-A$ must fail to be arcwise connected and hence by 8.2 indecomposable. Therefore $X$ is a Knaster continuum.

Suppose $X$ is a Knaster continuum and $A_{A B}$ an arc in $\mathcal{C}(X)$. Since $\sigma\left(\mathcal{A}_{A B}\right)$ is indecomposable by 8.1 we have $\sigma\left(\mathcal{A}_{A B}\right) \in \mathcal{A}_{A B}$. Hence the function $\mu$ assumes a unique maximum on any simple arc, and if $C=\sigma\left(\mathcal{C}_{A B}\right)$ then $\mu$ must be strictly monotone on each of $\mathcal{A}_{A C}$ and $\mathcal{A}_{C B}$. For $C_{1} \in \mathcal{A}_{\overline{A C}}$ we then have $C_{1}=\sigma\left(\mathcal{A}_{A C_{1}}\right)$. It follows that $A_{A C}$ and $A_{C B}$ are, with proper parametrization,

(14) The only known example of a continuum of this type was given by B. Knaster in his dissertation, Un continu dont tout sous-continu est indécomposable, Fundamenta Mathematicae vol. 3 (1922), pp. 247-286. 
segments. From 8.3 we see that there exists a unique continuum containing $A$ at which $\mu$ assumes any specified value. Hence the $\operatorname{arc} \mathcal{A}_{A B}$ is unique.

8.5. TheOREm. If $X$ is a Knaster continuum, for every $\epsilon>0$ there exists a monotone interior transformation $f(X)=Y$ such that $0<\operatorname{dia} f^{-1}(y)<\epsilon$ for all $y \in Y$.

Proof. Choose $d>0$ such that if $\mu(A)=d$ then dia $A<\epsilon$. For each $a \in X$ there is, by 8.4 , a unique $A(a) \in \mathcal{C}(X)$ such that $a \in A(a)$ and $\mu(A(a))=d$. If $A(a) \cdot A(b) \neq 0$ then by $8.3 A(a)=A(b)$. If $\lim a_{i}=a$, then since $\mu$ is continuous and $A(a)$ single-valued $\lim A\left(a_{i}\right)=A(a)$. The map $A(a)$ is then a continuous monotone interior transformation of $X$ into $\mathcal{C}(X)$ and satisfies the conditions of the theorem.

8.6. TheoRem. If $X$ is a Knaster continuum and if there exists, for every $\epsilon>0$, a monotone interior transformation $f(X)=Y$ such that:

(a) $0<\operatorname{dia} f^{-1}(y)<\epsilon$ for all $y \in Y$;

(b) $\operatorname{dim} Y<\infty$,

then $\operatorname{dim} X=1$.

Proof. Under the hypotheses of the theorem we shall exhibit an $\epsilon$-covering of order 2 of $X$ by closed sets. Choose $X_{0} \subset X$, by 7.8, closed, totally disconnected, with $f\left(X_{0}\right)=Y$. Let $U$ be an open set about $X_{0}$ so that the diameter of any component of $\bar{U}$ is less than $\epsilon$. Every component of $X-U$ is of diameter less than $\epsilon$, for if $A \subset X-U$, dia $A \geqq \epsilon$ then for $a \in A, f^{-1}(f(a)) \subset A$. But this contradicts the fact that $f\left(X_{0}\right)=Y$. Write each of $\bar{U}$ and $X-U$ as the sum of a finite number of closed disjoint sets of diameter less than $\epsilon$. The resulting covering of $X$ is surely of order 2 .

From 8.4 and 8.6 and the fact that the monotone image of a Knaster continuum is also a Knaster continuum we have:

8.7. THEOREM. If $X$ is a Knaster continuum of dimension greater than 1 then:

(a) for every $\epsilon>0$ there is a monotone interior transformation $f(X)=Y$ $0<\operatorname{dia} f^{-1}(y)<\epsilon$ for all $y \in Y$, with $\operatorname{dim} Y=\infty$;

(b) there exists an $\epsilon>0$ such that for any monotone interior $f(X)=Y$, with $0<\operatorname{dia} f^{-1}(y)<\epsilon$ for all $y \in Y$, it is true that $\operatorname{dim} Y=\infty$;

(c) there exist Knaster continua of infinite dimension.

Remark. Theorem 8.6 could be demonstrated without the restriction (b) on dimension if instead of 7.8 we had at at our disposal the theorem: If $f(X)=Y$ is monotone interior then there exists $X_{0}$, closed in $X$, with $f\left(X_{0}\right)=Y$, such that $X_{0} \cdot f^{-1}(y)$ is totally disconnected for all $y \in Y$; that is, such that $f$ is light on $X_{0}$. This statement is much weaker, except for restriction on $\operatorname{dim} Y$, than 7.8, and its truth would imply that every Knaster continuum is of dimension one. 


\section{BibliogRAPHY}

1. K. Borsuk and S. Mazurkiewicz, Sur l'hyperespace d'un continu, Comptes Rendus des Séances de la Société des Sciences et des Lettres de Varsovie, vol. 24 (1931), pp. 149-152.

2. K. Kuratowski, Topologie, p. 92.

3. S. Mazurkiewicz, Sur les continus absolument indécomposables, Fundamenta Mathematicae, vol. 16 (1930), pp. 151-159.

4. - Sur l'hyperespace d'un continu, Fundamenta Mathematicae, vol. 18 (1932), pp. 171-177.

5. - Sur le type C de l'hyperespace d'un continu, Fundamenta Mathematicae, vol. 20 (1933), pp. 52-53.

6. - Ein Zerlegungsatz, Fundamenta Mathematicae, vol. 23 (1934), pp. 11-14.

7. L. Vietoris, Kontinua zweiter Ordnung, Monatshefte für Mathematik und Physik, vol. 33 (1923), pp. 49-62.

8. T. Wazewski, Sur un continu singulier, Fundamenta Mathematicae, vol. 4 (1923), pp. 214-235.

9. M. Wojdyslawski, Sur la contractilitê des hyperespaces de continus localement connexes, Fundamenta Mathematicae, vol. 30 (1938), pp. 247-252.

10. - Rétractes absolus et hyperespaces des continus, Fundamenta Mathematicae, vol. 32 (1939), pp. 184-192.

UNIVERSITY OF VIRGINIA, Charlottesville, Va.

UNIVERSity OF NOTRE DAME, Notre DAME, IND. 